\title{
Ueber den Verlauf und die periphere Endigung des Nervus cochleae.
}

\author{
Von
}

\author{
Dr. med. J o hit a Kis hi, \\ Ohrenarzt aus Japan.
}

Hierzu Tafel VI.

Die Kenntniss des feineren Baues der Simnesorgane gehört im Allgemeinen zu den am besten durchgearbeiteten Capiteln der mikroskopischen Anatomie, denn sowohl zahlreiche Anatomen rom Fache wie auch Specialuntersucher aus practischen Berufen haben sich bestrebt unsere Einsicht in dieses schwierige Gebiet zu fördern. Eine besondere Stellung nimmt dabei das Gehörorgan eill. Auge und Nase bieten zunächst in so fern einfachere Verhältnisse dar, als sie relativ oberflächlich liegen und mit Leichtigkeit aus ihrer Umgebung ausgelöst werden können. Beim Gehörorgane bereitet jedoch gerade dieser erste Schritt zur Untersuchung schon bedeutende Hindernisse, denn dasselbe ist bekanntlich bei erwachsenen Thieren höherer Ordnung, insbesondere bei den uns speciell interessirenden Säugethieren, fest in eine knöcherne Capsel eingemauert. Schon allein das Herausmeisseln des (xehörorgans bietet techinische Schwierigkeiten und erlaubt die Untersuchung nur besonders Erfahrenen. Wohl mit aus diesem Grunde ist das Gehörorgan erst relativ spät gerade in seinen Feinheiten erforscht worden und haben seiten der Neurologen nur relativ Wenige Lust gezeigt sich eingehend mit diesem schwierigen Gebiete zu befassen.

Erst um die Mitte dieses Jahrhunderts klärte Corti den lau des Gehörorganes in seinen wesentlichen Zügen, und verewigte seinen Namen durch die Entdeckung des nach ihm benannten Organs. Nach Corti ist manche interessante Thatsache von 
Seiten der Anatomen an den Tag gefördert worden. Auch haben Physiologen, Pathologen und otologen sich in gleicher Richtung bemült; denn an die Kenntniss der Structur knüpft sich naturgemäss auch die Frage nach der Function dieses complicirten Organes. Nichtsdestoweniger bestehen auch noch jetzt Lïcken in unserem Wissen, welche insbesondere in der Otologie, meinem Specialgebietc, störend empfunden werden.

Als ich nach Europa kam, um im Laufe einiger Jahre unter Geheimrath Schwartze's Leitung eine weitere Ausbildung in meinem Fache zn erlangen, benutzte ich die Gelegenheit, gleichzeitig an dem Königlichen anatomischen Institute zu Halle auf breiter Basis mikroskopisch-anatomische Untersuchungen am Gehörorgane anzustellen. Es sei mir gestattet, auch an dieser Stelle dem Dircetor des anatomischen Instituts, Herrn Prof. W. Roux, sowie Herrn Prosector I’rof. E. Mehnert meinen Dank auszusprechen.

Zunächst wollte ich die feinere Structurirung des Gehörorganes, speciell der Schnecke bei den verschiedenen Säugethieren aus eigener Erfahırung kennen lernen. Ich untersuchte dieses Organ bei Kaninchen, Hunden, Katzen, Mecrschweinchen, Ratten, Schafen, Kälbern, Schweinen, Oclisen und, so weit es möglich war frisches Material zu bekommen, auch bei dem Menschen. In zweiter Linie war es meine Absicht mich in der Technik zu vervollkommnen. Ich wandte daher nach einander alle bisher vorgeschlagenen Fixations-, Fürbungs-, Einbettungs- und Nachbehandlungsmethoiden an und verglich die Methoden auf ihren Werth untereinander. Meine Untersuchungen sind zur Zeit noch lange nicht abgeschlossen; ich beabsichtige über die Ergebnisse zusammenfassend in einer grösseren Arbeit zu berichten. In vorliegender Abhandlung will ich nur einige von mir gewonnene Resultate mittheilen, welche sich auf den Verlauf des N. cochleae von seinem Durchtritte durch die Habenula perforata an bis zu seiner Endigung in oder an bestimmten Zellen beziehen; und zwar werde ich mich liauptsächlich auf die an Kaninchen und Hunden gewonnenen Resultate beschränken und die ahnlichen Befunde an anderen Süugethieren und an Menschen nur zum Vergleiche heranziehen.

Auf die bei meinen Untersuchungen angewandte Methode will jch hier nicht nüher eingehen, weil ich sie später (s.S.30u.f.) darübe mir 
Zusammenhang ausführlich mitzutheilen gedenke. Ich bemerke hier nur, dass ich für die Untersuchung der Endigungsweise der Nervenfasern in der Haarzelle eine Hämatëinfärbung in neuer, von Apathy abweichender Weise angewendet habe.

\section{Arten der Zellen, welche den inmern Abhang der Papilla spiralis bilden.}

Bevor ich meine Ergebnisse über die Verlaufsweise der peripheren Endfasern des Nervus cochleae zur Darstellung bringe, halte ich es für nothwendig, mich zunächst über die Beziehungen zu aussern, in welchen die durch die Löcher der Habenula perforata kommenden feinen. nackten Axencylinderfasern, die vor dem Durchtritte ihr Mark und die Schwann'sche scheide verloren haben, zu jener Zellengruppe stehen, die sie zunächst passiren. Diese ist von den Autoren mit verschiedenen Namen: Körnerschicht (Waldeyer, Gottstein), unter- innere Deckzellen. (Henle), subepitheliale Schicht (Schwalb) belegt worden. Ihnen liegen nach innen noch epitheliale Zellen an, welche Retzi us als innere Stützzellen, Krause als innere Deckzellen bezeichnet. Diese bilden mit den Zellen der vorigen Gruppe und mit den inneren Haarzellen einen Wulst, den Schwalbe auf dem Querschnittsbilde „Epithelialdreieck an der inneren Abdachung des Corti'schen Organs" benennt. Wir haben uns also zunächst über diese Zellen zu orientiren.

Ueber diese Zelleugruppe schrieb zuerst Deiter s ${ }^{1}$ ) im Jahre 1860 in seinen „Untersuchungen über die Lamin spiralis membranacea", dass er an der Innenseite des Bogens, ausser einer Reihe cylindrischer, cilientragender Zellen, noch ein System von kleinen, theils rundlichen, theils spindelförmigen Zellen, welche durch ihre anastomosirenden Ausläufer ein Faserzellen - Netz bildeten, gefunden habe. Aber über ihre leziehungen zu den Nervenfasern machte er keine nähere Angabe.

Nach ihm veröffentlichte Böttcher,$^{2}$ ) der Entdecker der inneren Haarzellen, neue Beobachtungen über unsere Zellen. Er fand unterhalb der inneren Haarzellen zwei kleine Zellen mit Bomn. 1860.

1) B. Deiters, Untersuchungen übcr die Lamina spiralis membranacea.

2) Böttcher, Ueber Entwicklung und Bau des Gehörlabyrinthes nach Untersuchungen an Süngethieren. Dresden 1869. 
kleinerem Kern, dic mit ihren oberen, durch einen Spalt von einander geschiedenen Theilen, direct mit den Haarzellen in Verbindung stehen, während sic mit einem unteren Fortsatze auf der Membrana basilaris befestigt sind, mit einem anderen unteren Fortsatze sich gegen die Habenula perforata wenden und mit den Nervenfasern sich verbinden. Diese 'Thatsache erörterte er dann von Neuem in seinen "Kritischen Bemerkungen"1) und stellte vom Standpunkte der Fintwickelungsgeschichte aus fest, dass die Zellen mit den Nervenfasern zusammenlängen.

Als die Autoren aber, die diesen Zellen ganz besondere Aufmerksamkeit widmeten, sind Wald e y e ${ }^{2}$ ) und Got ts te in ${ }^{3}$ ) zu nemnen. Diese beiden betrachteten diese Zellen absolut als Nervenapparat, und nannten sie „innere Körnerschicht“. Wenn nun auch beide ïber die Beziehungsweise der Nerven etwas verschiedene Ausdriicke gebrauchten, waren sie doch in der Annahme einig, dass die Zellen oder ihre Auslaufer mit den Nervenfibrillen gewissermassen in Verbindung ständen.

Eine ganz abweichende Ansicht vertraten zwei andere Autoren; nämlich Lavdowsky ${ }^{4}$ ) und $\mathrm{Nuel}^{5}$ ), die die Körnerschicht als nicht mit den Nervenfasern in Verbindung stehend betrachteten. Jener behauptete, der Bau der Körnerschicht sei ziemlich complicirt; sie bestehen aus mindestens drei Theilen, zwei faserigen und einem zelligen; die Zellen seien grosse runde Korperchen oder besser entwickelte Kerne mit relativ sparsamer körniger, feiner Masse; Fortsätze an ihnen würden nicht beobachtet, dagegen zweierlei Arten von Fasern, von denen die einen Nerven, die anderen aber scharf wellige, den Basilarfasern parallele, nicht anastomosierende, nicht nervöse Fasern zweifelhafter Bedeutung seien, und die Radialnerven gingen durch diese Kornerschicht, aber wie es scheine, ohne sich mit deren Elementen

1) B öttcher, Kritische Bemerkungen und neue Beiträge zur Literatur der Gehörschnecke, 1)orpat 1872.

2) Waldeyer, Hörnerv und Schnecke, Handbuch von v. Stricker. Leipzig 1872.

3) Gottstein, Ueber den feineren Bau und die Entwickelung der Gehörschnecke bei Menschen und Säugethieren. Bonn. 1871.

4) Lavd ow sky, Untersuchungen úber den akustischen Endapparat der Säugethiere. Archiv f. mikrosk. Anat. Bd. 13. 1876.

s) $\mathrm{N}$ u e l, Recherches miroscopiques sur !'anatomie du limaçon des mammifères. 1878. 
zu vereinigen. Nuel schrieb einfach, die durch die Habenula perforata tretenden Fasern gingen zwar zwisclen den Zellen der Körnerschicht hindurch, ob sie in năherem Verhältniss ständen, sei zweifelhaft.

Nun gab Retzius ${ }^{1}$ ), indem er die bis zu seiner Zeit veröffentlichten Forschungen über das Gehörorgan einer sehr genauen Beurtheilung unterwarf, auch über diese Zellen der Körnerschicht seine Ansicht ab. „Körner", so schrieb er, "will ich dieselben nicht nennen, da dieser Name vielleicht angiebt, dass sie zum nervösen Apparat zu rechnen seien, und ich sie zum indifferenten Epithel zähle." Er sah die \%ellen also nicht als zum Nervenapparat gehörig an, sondern rechnete sie zu den Fadenzellen und nannte sie innere Stützzellen.

Diesem Urtheil des berühmten Forschers, der sich um den Fortschritt der Anatomie des Gehörorganes die grössten Verdienste erworben hat, muss ich indess gleich $\mathrm{Sch}$ w alb $\mathrm{e}^{2}$ ) entgegentreten, der in seinem Lehrbuch der Anatomie des Ohres sich folgendermassen aussert: "Meiner Ansicht nach muss man diese Lage (Körnerschicht) wohl von den vorhin erwihnten inneren Stützzellen trennen Sie ist durch die erste Ausbreitung der durch die Foramina nervina in das Corti'sche Organ eintretenden Nervenfasern characterisirt, und mir will es scheinen, als werde sie hauptsächlich durch diese Nervenfasern gebildet und gehören die Kerne zu den Nerven, vielleicht als Kerne von Gliazellen, ähnlich denen, welche in so grosser Zahl in der marklosen Nervenfaserschicht der Retina sich finden."

Auch sonst noch haben viele Autoren, wie Tafani, Katz, Steinbrügge, Siebenmann u A. Schriften über die Endfasern des Nervus cochleae veröffentlicht; doch haben sie auffallender Weise diesen Gegenstand sehr wenig berïcksichtigt.

Um nun dem Wesen dieser Zellen näher zu kommen, scheint es zweckmässig, der Entwickelungsgeschichte derselben etwas Aufmerksamkeit zu schenken, was allerdings schon Baginsky ${ }^{3}$ ) gethan. Dieser nämlich sagte: „Hier glaubt man holm 1884 .

1) Retzius, Das Gehörorgan der Wirbelthiere. Bd. II. Stock-

2) G. Schwalbe, Lelurbuch der Anatomie des Ohres. S. 379. Erlangen 1887.

3) Baginsky, Zur Entwickelung der Gehörschnecke. Archiv für mikrosk. Anatomie. 1886. 
Ueber den peripheren Verlauf und die Endigung des Nervus cochleae. 149

bei oberflächlicher Betrachtung in der That Bilder zu haben, wie sie Boettcher beschrieben hat. Bei genauerer Untersuchung indes überzengt man sich, dass diese Zellen mit den inneren Haarzellen gar keinen Zusammenhang haben, dass vielmehr die innere ibren Fortsatz medial, die cuussere lateral von den inneren Haarzellen nach oben gegen die obere Begrenzung des grossen bezw. kleinen Fithelialwulstes sendet. Und ganz besonders auffallend ist das Verhalten dieser lateral von der inneren Haarzelle gelegenen Zelle; sie zeigt nämlich in allen weiteren Eutwickelungsstadien das nämliche Verhalten, wie wir es an den Deiters'schen Zellen sehen, sodass wir sie als eine denselben homologe Bildung betrachten können und sie aus den von Retzius und Schwalbe angegebenen inneren Stützzellen noch besonders hervorheben müssen." Ueber das Verhältniss dieser Zellen zu den Nerven erwähnte er jedoch nichts.

Nach meinen an verschiedenen Thieren gemachten Beobachtungen nun habe ich in dem Embryonalstadium, wo ich in dem noch kleinen Epithelialwulst schon verschiedene Zellen habe unterscheiden können, wie auch später in dem grossen Epithelialwulst unterhalb der inneren Haarzellen unter den einfachen embryonalen Epithelzellen, die ich kurz als "Embryonalzellen" bezeichnen will, zwei verschiedene Arten von anderen Zellen gesehen; die eine (siehe Fig. $1 \mathrm{e}, \mathrm{g}$ ) ist blass und in den meisten Fällen nur in der Finzahl vorhanden und hat einen grossen runden, die andere durch zwei Zellen, deren eine nach innen, die andere nach aussen der vorigen liegt, hat einen langovalen Kern. Und diese zwei Arten von Zellen bleiben während der Fortdauer der Entwickelung des Embryo immer in derselben Gegend, zwischen den unteren Enden der inneren Haarzellen und der Lamina membranacea, erhalten, während die anderen Embryonalzellen durch Atropbie allmählich zum grössten Theil verschwinden. Hierbei erscheinen in der Umgebung der Zellkörper viele Nerventibrillen, die aus den Foramina nervina hervorkommen. Diesen Zustand kann man an vor zwei Tagen geborenen Kaninchen ganz deutlich bemerken, wie Figur 2 zeigt. Während der grosse Epithelialwulst schon etwas verschwunden und der Sulcus spiralis internus zum Theil von einschichtigem Epithel bekleidet ist, finden sich doch noch viele atrophirte Embryonalzellen, deren Zellkörper sehr faserartig verändert sind; 
und besonders steigen dicht neben den inneren Haarzellen merkwürdig viele Fasern empor, und ausserbalb dieser Zellen sind ganz deutlich die zwei Arten Zellen (eg und is) zu bemerken. Bei den eine Woche alten Kaninchen verschwinden die Embryonalzellen schon etwas mehr; nach $2-3$ Wochen wird ihre Zahl noch geringer und bei Erwachsenen finden sich nur einige Zellen, welche den Epithelzellen des Sulcus spiralis internus gleichartig sind. So scheint es mir, dass der faserreiche Zustand dieser Zellenschicht einReductionsstadium der embryonalenEpithelzellen darstellt.

Was nun die zwei Arten von Zellen betrifft, die wir oben von den sich rückbildenden Embryonalzellen unterschieden haben, so entsendet diejenige, welche einen ovalen Kern hat, bei eine Woche alten Kaninchen vom Zellkörper aus nach oben entweder einen einfachen glatten oder einen mit faserigen Protoplasmaresten behafteten Fortsatz zu dem Cuticularsaum der inneren Haarzellen hin, und nach unten zu einen Fortsatz, der in schräger Richtung nach der Umgebung der Foramina nervina der Lamina membranacea hinüber lauft.

Die andere Zelle, die mit dem runden Kern, steht schon in früher embryonaler Zeit in intimerem Verhaltnisse zu den Nervenfasern. Doch kann man zu der Zeit, wo der Epithelialwulst ein wenig reducirt worden ist, die näheren Verhältnisse dieser Zellen zu den Nervenfasern noch nicht genau bemerken.

Bei dem $8 \mathrm{~cm}$ langen Kaninchenembryo, bei welchem die Veränderung des grossen lepithelialwulstes noch nicht lange angefangen hat, kann man nur die Verbindung dieser Zellen mit den Nervenfasern, die durch die Foramina nervina durchgetreten sind, beobachten. Bei einem vor zwei Tagen geborenen Kaninchen, bei dem der grosse Epithelialwulst etwas geschwunden ist, tritt aber die Verbindungsweise mit den Nervenfasern schon deutlich zu Tage.

Obgleich in diesem Stadium die Zellkörper der ,Embryonalzellen" durch Reductionsprocesse sehr faserreich geworden und den mit den Nerven in Verbindung stehenden Zellen sehr ähnlich sind, kann man doch beide Arten dadurch sehr leicht unterscheiden, dass die "Embryonalzellen" durch ihren unteren Fortsatz innerhalb der Foramina nervina mit der Lamina membranacea zusammenhängen, aber niemals mit den durch die Foramina nervina durchtretenden Nervenfasern verbunden sind. 
Von den Zellen, die mit den durch die Foramina nervina durchtretenden Nervenfasern verbunden sind, finden sich in einem radialen Schnitte gewöhnlich eine oder zwei; in letzterem Falle steht die eine immer dicht an den inneren Pfeilerzellen und ungefähr im Mittelpunkte des Raumes zwischen dem Ende der inneren Haarzellen und der Lamina membranacen, die andere gewöhnlich etwas oben und nach innen von jenen Haarzellen; beide sind nach unten, wie deutlich $\mathrm{zu}$ erkennen ist, mit den Nervenfasern verbunden. Darum scheint es mir, dass die durch die Löcher der Lamina membranacea herankommenden Fasern nur mit diesen Zellen in Verbindung stehen. Von diesen Zellen gehen dann nach oben $\mathrm{zu}$ den inneren Haarzellen die varicösen Fasern und nach aussen ebensolche Fasern durch die Zwischenrïume der inneren Pfeilerzellen. Auch durch seitliche Fortsătze scheinen sie miteinander verbunden zu sein.

Bei den erwachsenen Thieren, bei denen die „Embryonalzellen" ganz verschwunden sind, unterscheide ich hier drei Arten Zellen: 1. die Zellen, die mit den Nervenfasern verbunden sind; sie haben einen runden oder schwach ovalen Kern und keinen glatten Fortsatz; sie allein stehen durch die Nervenfasern, die durch die Foramina nervina hindurchkommen, mit der Lamina spiralis membranacea in Verbindung; 2. die Zellen, die nach Retzius u. A. reducirte Zellen der Embryonalzellen sein sollen und "innere Stützzellen" genannt worden sind; sie haben einen sehr schmalovalen Kern und senden davon nach oben und unten einen glatten, runden Fortsatz; 3. die Zellen, die ganz gleichartig den Zellen des Epithels des Sulcus spiralis internus sind. Ich fasse meine Ansicht dahin zusammen, dass die letzteren Zellen allein den Rest der reducirten "Embryonalzellen“ darstellen, und dass die beiden anderen Zellenarten nicht von solchen reducirten „Embryonalzellen "stammen, sondern ebenso wie die Haarzellen im Embryo eine besondere Anlage besitzen. In früherer Zeit des Embryo sind sie aber von den spater reducirten Embryonalzellen noch nicht besonders zu unterscheiden, sie treten erst mit dem Verschwinden dieser Zellen immer deutlicher in ihrer Besonderheit hervor. Diejenigen Zellen, welche mit Nervenfasern verbunden sind, sehe auch ich ihrem Wesen nach als eigenthümliche Ganglienzellen an nnd möchte sie wie Waldeyer und Gottstein mit den subepithelialen Ganglien- 
zellen der inneren Körnerschicht der Retina vergleichen, während $\mathrm{Schwalbe}$ einen Vergleich mit Gliazellen für zutreffender hält; die anderen Zellen, die mehr schmalovalen Kern haben, sind wohl mit Recht innere Stützzellen zu nennen, wie Retzius und Schwalbe bereits gethan haben.

\section{Verlauf der peripheren Endfasern des Nervus cochleae.}

Obgleich bereits viele Forscher vor Deiters die Verlaufungsweise der Endnervenfasern untersucht baben, ist doch der wahre Thatbestand erst von ihm bemerkt worden. De it ers') unterschied nämlich unter den Endnervenfasern zwei Systeme, ein longitudinal und ein transversal verlaufendes; die longitudinalen Fasern zertheilen sich wieder nach zwei verschiedenen Richtungen, von deneu die einen zu den aufsteigenden Corti'schen Fasern hinaufgehen, die anderen unter den Corti'schen Bogen treten und zwar hier entweder der Basilarmembran anliegend weiter verlaufen oder an der unteren Fläche der Corti'schen Faserzellen aufsteigen. Die transversalen Fasern gliedern sich auch in mehrere Bündel. Das erste liegt ungefähr unter und in der Mittelhöhe der aufsteigenden C o r t i'schen Fasern ; ein zweites liegt unter der Berührungsstelle der beiden Faserreihen. Fin drittes, weniger constantes, findet sich bisweilen an der Innenfläche der Fasern zweiter Ieihe oberhalb der Glocken. Ein viertes Bündel verläuft an der inneren Seite der Verbindungsstiele. Wenn nun Deiters auch die Aufmerksamkeit auf die transversalen Züge lenkte, vermochte er doch nicht zu sagen, was aus diesen queren Fasern werde, er war der Meinung, dass die Fasern des queren Systerns aus dem longitudinalen System ihren Ursprung nehmen.

Nach Deiters veröffentlichte Kölliker ${ }^{2}$ ) in der fünften Auflage seiner Gewebelehre eigene Untersuchungen, wobei er sich im Ganzen vor Allem Deiters anschloss; Kölliker unterschied nämlich auch Querzüge und Längszüge. Jene, die er Fibrae transversales nannte (F. longitudinales Deiters) sind die

') Deiters, Untersuchungen über die Lamina spiralis membranacea. Bonn. 1860.

2) Kölliker, Handbuch der Gewebelehre des Menschen. Fünfte Auflage. Leipzig 1867. 
unmittelbaren Fortsetzungen der aus den Löchern der Habenula perforata kommenden Fasern, und zerfallen in zwei Abtheilungen. Die Längszüge nannte Kölliker Fibrae longitudinales (F. transversales Deiters), und wies sie bei den Menschen und Ochsen an drei verschiedenen Stellen nach: 1. unterhalb der Mitte der inneren Corti'schen Fasern, 2. unterhalb der Verbindung der beiderlei Corti'schen Fasern, 3. unterhalb der äusseren Corti'schen Fasern. Bei der Katze sah er solche Fasern sicher nur: 1. zwischen der Mitte der äusseren Corti'schen Fasern und der ersten Reihe der Corti'schen Zellen, 2. zwischen der ersten und zweiten Reihe der Corti'schen Zellen, 3. zwischen der zweiten und dritten Reihe der Corti'schen Zellen.

Nächst diesen zwei Autoren verdient Loewenberg ${ }^{1}$ ) genannt zu werden, der zuerst darauf aufmerksam machte, dass die Nervenfasern des Corti'schen Organs theils radiär, theils spiralig verlaufen. Loewenberg fand unter den radialen Fasern vier verschiedene Arten: 1. die Fasern, welche in den Tunnel eintreten, um sich mit einem, in der Mitte zwischen den inneren und den äusseren Basilarzellen liegenden Körperchen zu vereinigen; 2. die Fasern, welche in dem Tunnel zu einem an der oberen Vereinigung der Corti'schen Pfeiler liegenden Körperchen gehen; 3. die Fasern, welche aus dem erstgenannten Körperchen heraustreten und nach aussen hinlaufen, um an der Lamina reticularis oder an den Zellen derselben zu endigen; 4. die umliegenden Fasern, welche sich nach innen hin zu dem Sulcus spiralis internus umbiegen. Loewenberg wies auch schon nach, dass die spiraligen Nervenfasern beim Menschen zahlreicher und leichter zu finden sind als bei den Thieren. Beim Menschen sind in der Hauptsache im Innern des Corti'schen Tunnels zwei Arten von Bündeln vorbanden: 1. an der Mitte der inneren Pfeiler; 2. an den äusseren Pfeilern, über ihrem basilaren Ende. Auch wollte Loewenberg sonst noch ausserhalb des Corti'schen Tunnels ein Bündel Fasern derselben Art zwischen der Mitte der äusseren Pfeiler und der ersten Reihe der Corti'schen Zellen sowie noch ein weiteres Bündel an der Mitte der üusseren Pfeiler gefunden haben.

1) Loewenberg, La lame spirale du limaçon de l'oveille de l'homme et des mammifères. Journal de l'anatomie et la Physiol. 1868. 
Unter den weiteren Autoren sind noch zu nennen: Gottstein ${ }^{1}$, Waldeyer ${ }^{2}$ ) und Nuel. Die beiden ersten unterschieden ebenfalls radiale und spirale Faserzüge. Die radialen Faserzüge, die im Zwischenraume der zwei inneren Pfeiler etwa durch die Mitte der Corti'schen Bogen bis zu den ausseren Haarzellen verlaufen, bezeichnen diese Untersucher als „aussere radiale Fasern", und die Fasern, die von den Löchern der Habenula perforata direct bis zu den inneren Haarzellen herantreten, nennen sie "innere radiale Fasern". Unter den spiralen Faserzügen unterscheiden sie auch „einen inneren und einen äusseren Zug" ; der innere und zugleich schwächere Zug entspricht der Reihe der inneren Haarzellen, der starke, äussere, in drei parallele Abtheilungen gegliederte Zug den drei Reihen der äusseren Haarzellen.

$\mathrm{Nuel}^{3}$ ), der ungefähr dieselbe Ansicht wie Waldeyer vertritt, sagte von den Nervenfasern: Im Tunnel verlaufen manche spiral wohl unter 60 äusseren Bogen hin; andere verlaufen mehr schräg; einige nähern sich der radiüren Richtung, ohne wirklich radiär zu werden. Ausserdem sah $\mathrm{Nuel}$ ebenso wie Waldeyer ein System paralleler spiraler Fasern an den Stielen der inneren und äusseren Haarzellen, welche viel dünner und feiner als die Nervenfasern unter den C orti'schen Bogen sind, wollte dieselben aber nicht als Nervenfasern ansehen; auch zwischen den äusseren Stützzellen sah $\mathrm{Nuel}$ ein System solcher spiraler Fasern.

Obgleich die Ansichten der Forscher, die ich bisher erwähnt habe, in verschiedenen Punkten von einander abweichen, sind sie doch in der Hauptsache einig. Wir können danach als gesichert annehmen, dass es unter den Endnervenfasern des Nervus cochleae zwei verschiedene Fasersysteme giebt, die als die transversalen und die longitudinalen oder als die radialen und die spiralen Nervenzüge bezeichnet worden sind. Es fanden sich aber anderseits auch damals bekannte Autoren, welche das Vorhandensein der Spiralzüge in Abrede stellten, nämlich Middendorf und Boettcher.

1) Gottstein, l. c.

2) Waldeyer, l. c.

3) Nuel, Beitrag zur Kenntniss der Säugethierschnecke. Archiv für mikroskop. Anatomie. 1872. 
B oettcher ${ }^{1}$ ) schrieb: „Die longitudinalen Fasern werden nicht eher anerkannt werden, als sie auf dem Durchtritt demonstrirt sein werden. Das hat aber noch Niemand gethan, ja, es hat an einem solchen sogar noch Niemand die Stelle angeben können, wo sie liegen." Das Vorhaudensein dieser beiden Endnervenfasern wurde durch die genaueren Untersuchungen von anderen Forschern immer weiter gesichert. Insbesondere sind auch hier unter den Autoren Retzius, Hensen, Lavdowsky und $\mathrm{Nuel}$ zu nennen.

Hensen ${ }^{2}$ ) erklärte zuerst in seiner 1873 veröffentlichten Besprechung, dass ihm das Vorhandensein der longitudinalen Stränge der Nerven nun nicht mehr zweifelhaft sei. Er sagte, dass diese Nerven eigentlich als Nervenplexus bezeichnet werden müssten, von dem ein innerster longitudinaler Nervenzug nach innen von dem inneren Pfeiler, ziemlich dicht neben diesem, in dem Epithelrest des grossen Wulstes liegt; die zweiten longitudinalen Nervenzüge liegen diesem gegenüber ein wenig tiefer. Die beiden Faserzüge werden verbunden durch kurze Querstränge, welche zwischen je zwei inneren Pfeilern hindurchgehen. Von dem äusseren der beiden Züge gehen dann Fasern quer durch den Tunnel, in einer den äusseren Pfeilern entsprechenden Zahl, zwischen diesen oberhalb ihrer Füsse hindurch, um einem dritten longitudinalen Nervenzweige anzuliegen.

Lavdowsky ${ }^{3}$ ) unterscheidet unter den Endnervenfasern die Radial- und Spiralnerven, und theilt jene wieder in die inneren und ausseren Radialnerven. Die inneren Radialnerven gehen zu den inneren Endzellen, und die äusseren Radialnerven dringen durch die acustische Körnerschicht, ohne sich mit deren Elementen zu vereinigen, zwischen je zwei Pfeilern hindurch und theilen sich in zwei Schichten. Die eine von diesen verläuft geradeaus und sehr wenig oder nicht verzweigt, ungefähr nach der mittleren Höhe der äusseren Bogen, um alsdann in die Reihen der äusseren Endzellen einzudringen; während die andere Schicht nit verastelten und getheilten Fasern fast parallel mit dem Tumnelboden läuft, frei über der Membran gelagert, und in einzelnen Regionen

1) Boettcher, Kritische Bemerkung und neue Beiträge zur Literatur des Gehörlabyrinthes. Dorpat. 1872.

z) Hensen, Besprechung. Archiv für Ohrenhellkunde. 1873.

3) Lavdows ky l. c. 
sich seitwärts wendet. Diese letzteren hat Lavdowsky als $\mathrm{S}$-artig gebogene Radialnerven bezeichnet. Ueber die Spiralnerven sagte er, dass sie, besonders diejenigen unter ihnen, welche zu den inneren Endzellen gehören, bedeutend dünner als die Radialnerven seien, und dass die anderen drei Züge der ausseren Spiralnerven stets ejnander parallel durch alle drei Windungen hinzögen, nämlich der erste $\mathrm{Zug}$ zwischen den ersten und zweiten Corti'schen Zellen, der zweite zwischen den zweiten und dritten $\mathrm{C}_{0}$ rti'schen Zellen, der dritte zwischen den dritten Corti'schen Zellen und den Hensen'schen Stützzellen.

$\mathrm{Nuel}^{1}$ ) stellte auch Untersuchungen an über die Schnecke des Hundes und der Katze und bestätigte das Vorhandensein der spiralen Nervenfasern im Tunnelraum. Ausserdem fand er beim Hunde noch eine besondere Art von Nervenfasern, am oberen Ende des Schneckencanales, in grosser Menge. Diese behalten ihre Markscheide noch im Tunnelraum, ja sogar bis zu den äusseren Pfeilern; sie zeigen im Tunnel eine spirale Richtung, in dem sie schief nach aussen gehen und geben plötzlich ihre Markscheide ab und setzen dann ihren spiralen Verlauf weiter fort, bis sie zwischen den äusseren I'feilern heraustreten. Mehr als die Hälfte der Fasern verliert jedoch ihre Markscheide schon vor dem Eintritt in den Tunnel. Nuel erklärte ferner, er habe gegen die Basis der Schnecke des Hundes und der Katze keine Spiralfasern mehr gesehen, weshalb er auch annimmt, dass alle Fäserchen hier in radialer Richtung den Tunnel durchziehen. Beim Kaninchen, Meerschweinchen und Schaf fand Nuel, dass alle Nervenfäserchen als blasse Fasern in radialer Richtung den Tunnel durchgingen. Ausserhalb des Tunnels fand Nuel zwei verschiedene spirale Nervenfasersysteme. Das eine findet man in der Gegend der Cylinder der De iters'schen Zellen; es ist dies ein System spiraler, ziemlich kräftiger, varicöser Fasern, welche, unter einander parallel, zuerst allmählich, dann schneller gegen die Corti'schen Zellen emporsteigen. Wenn man dann den Tubus etwas senkt, erblassen die Fadenfortsätze der Corti'schen Zellen sowie die varicösen Fasern, und man sieht die Cylinder der Deiters'schen Zellen mit ihren unebenen Contouren. $\mathrm{Zu}$ gleicher Zeit tritt ein System äusserst feiner, nie varicöser Fasern

') $\mathrm{Nu}$ el, Recherches microscopiques sur l'anatomie du limaçon des mammifères. 1878. 
entgegen, welche ganz spiral, nie nach oben zu den Corti'schen Zellen und nie nach unten in der Nahe der Membrana basilaris verlaufen. Ijiese Fasern bilden das zweite System.

Retziusi) hat in seiner grossen Arbeit die Verlaufungsweise der Endnervenfasern der Schnecke der Kaninchen, Katze und des Menschen sehr genau vergleichend beschrieben. Nach seinen Angaben losen sich die Nervenfasern, nachdem sie nackt aus den Löchern der Canälchen der Habenula perforata ausgetreten sind, in schmale Fibrillenbündel auf, welche zwischen den unteren Enden der inneren Stützzellen emporsteigen und zum allergrössten Theil in den von Hensen beschriebenen innersten spiralen (longitudinalen) Strang cintreten: Beim Kaninchen sah Retzius nach oben zum Bereich der inneren Haarzellen emporsteigende feine varicöse Nerven, welche das untere Ende dieser Zellen umschweiften. I)ieser imerste spirale Strang erscheint im Querschnitt als ein ovaler Körper, welcher sich durch den Glanz von den nahe liegenden Kernen der Epithelzellen unterscheidet; oft aber erscheint er dümm und nicht besonders scharf abgrenzbar, weil seine librillen nicht ong an cinander gedrängt, sondern mohr aufgelöst und isolirt verlaufen. Von demselben gehen durch die sclimalen unteren Spalten zwischen den inneren l'feilerzellen kurze Nervenbündel ab, welche zu dem an der äusseren Fläche der inneren I'feilerzellen, nahe an ihren Füssen und dicht über ihren Kernen, hinstreichenden, lüngst bekannten spiralen Nervenstrang verlaufen und schliesslich sich in ihn einsenken. Dieser Strang, der nach seiner Meinung mit Recht der Tunnelstrang heissen könnte, war etwas dicker als der innerste Strang, im Querschnitte gewöhnlich oval oder rundlich-oval. Von ihm gehen in ganz kurzen Zwischenräumen die längst bekannten, radialen Fasern ab, welche sich etwas erheben und ziemlich gerade nach aussen zwischen die äusseren Pfeiler verlaufen; sie könnten die radialen Tunnclfasern gcnannt werden. Nachdem sie die äusseren Ifeilerzellen passirt, tauchen sie in jene complicirte Partie der Papilla basilaris ein, die unter den ausseren Haarzellen liegt. Retzius sah auch schon um die l)eiters'schen Zellen, 12-16 Reihen glïnzender Punkte, welche oben vom unteren Ende der Haarzelleu herab bis in die Nähe der Basilarmembran vertheilt sind. Diese Punkte halten drei Züge ein; der erste Zug liegt 1) Retzius, l, c. 
zwischen den äusseren Pfeilerzellen und den ersten Deit ers'schen Zellen, der zweite zwischen den ersten und zweiten Deitersschen Zellen, der dritte zwischen den zweiten und dritten Deiters'schen Zellen. Retzius konnte diese Punktziige bei manchen Isolationspräparaten sehr deutlich nachweisen und überzeugte sich hierbei sowohl von ihrer Zusammensetzung aus Fäserchen als von ihrer Aehnlichkeit mit wahren Nervenfäserchen. Retzius beobachtete ferner, dass die den Tunnel durchziehenden und zwischen die äusseren l'feilerzellen verlaufenden Radialfüserchen sich zu den oben beschriebenen spiralen Fäserchen umbiegen. Diese Thatsache konnte er in mehreren Pröparaten ganz unzweideutig und sicher constatieren; selten aber gelang es ilım, den Verlauf der Radialfasern zu den beiden äusseren Zügen zu sehen.

Bei der Katze fand Retzius, dass die durch die Habenula perforata durchkommenden nackten Fasern sich schnell in körnige Fibpillen auflösen; ein Theil dieser Fibrillen biegt sich nach der Seite hin um und verläuft in spiraler Richtung weiter, den ersten oder inneren Zug der Spiralfasern bildend. Theils von diesen Spiralfasern, theils direct von den aus der Habenula perforata austretenden Fasern steigen varicöse Fasern zu den unteren Enden der inneren Haarzellen empor. Von diesem inneren Spiralfaserzug treten zwischen den inneren Pfeilerzellen radialverlaufende Fasern in den 'Tunnel hinein, in dem sie wie beim Kaninchen, einen Spiralfaserzug an der Aussenseite der inneren Ifeilerzellen, den sogenannten zweiten spiralen oder Tunnelfaseryug bilden. Von diesem spiralen Zuge gehen dann die bekannten radialen Tunnelfasern nach aussen hin, indem sie sich gewöhnlich etwas erheben, und durch den Tunnelraum zwischen je zwei äusseren Pfeilerzellen, radial die immere Abtheilung des Nuel'schen Raumes durchziehen und mehr oder weniger hoch zu den Deiter'schen Zellen herantreten. Aber, sagte Retzius, "nicht selten nehmen sie jedoch einen anderen, weniger geraden Verlauf durch den Tunnelraum, indem sie sich zum Tunnelboden senken, sich ungefähr an der Grenze der Fussplatten der inneren und ausseren Pfeilerzellen anheften, hier zuweilen entweder den ganzen oder einen accessorisch spiralen Zug bilden und sich damn wieder erheben, um zwischen den ausseren Pfeilerzellen radial zu den Deiter'schen Zellen zu treten. An der immeren Seite der Deiters'schen Zellen augelangt, biegen sich alle diese Radialfasern wirklich um und verlaufen dann spiral." 
Die allgemeine Verlaufungsweise der Endnervenfasern beim Menschen entspricht nach seinen Beobachtungen nicht der bei Kaninchen und Katze. Iagegen fand Retzius, dass der Tunnelfaserzug, welcher in spiraler Richtung umbiegt, auf dem Querschnitte als scharf begrenzter, rundlicher oder ovaler Strang den ganzen Tunnel entlang zieht. Ferner findet sich ungefähr in der Mitte der inneren Seite der Reihen der Deiters'schen Zellen je ein äusserer spiraler Zug (also drei bis vier Züge), welcher aus einer dicht gedrängten Masse von parallel neben einander verlaufenden, feinen varicösen Fäserchen besteht; diese Fäserchen liegen beim Menschen in der Regel viel dichter beisammen und bilden auf dem Querschnitt ein länglich ovales Bündel, welches sich der Innenseite der Körper der Deiters'schen Zellen anschmiegt und nach oben hin bis zum unteren Ende der ausseren Haarzellen reicht. Das erste dieser Bündel liegt an der inneren Seite der ersten Reihe der Deiters'schen Zellen; das zweite, dritte und vierte liegt zwischen den betreffenden Deiters'schen Zellen zweiter, dritter und vierter Reihe. Zuweilen und zwar besonders in der Basilarwindung, liegen jedoch die Fasern nicht so dicht beisammen, sondern etwas von einander getrennt, in dünner Reihe neben den Deiters'schen Zellen, in gleicher Weise wie bei Kaninchen und Katze, und man trifft dann ibre körnchenähnlichen Durchschnitte noch tiefer, bis in die Nähe der Basilarmembrane.

T a f a $\mathrm{i}^{1}$ ), der hauptsächlich an Affen seine Untersuchungen angestellt hat, sagte, dass die Nerven, so wie sie die Habenula perforata verlassen hătten, die Markscheide verlören, dann längs der inneren Flache der inneren Pfeiler nach deren Fuss zögen, und sich darauf $z u$ einem einzigen laangsbündel vereinigten. Sie geben allmähliche seitliche Füden ab, welche sich fast immer in den untersten Theil der Iängsfasern zwischen den inneren Ifeilern eindrüngen. An der ausseren Fläche der inneren Pfeiler bilden die Fasern wieder ein kleines Bündel, von dem in bestimmten Intervallen radiale Fäden abgehen. Im Corti'schen 'Tunnel steigen die lasern also von der Basilarmembrane aus nach aussen aufwärts. Die Nerven treten an die Corti'schen Zellen gerade dort, wo sie von den Deiters'schen Zellen umfasst werden. Noch

1) Ta fani, L'organe de Corti chez les sings. Archiv. ital. de biologie 1885 . 
etwas tiefer gelegen als diese Fasern, zieht eine andere Serie von der inneren Fläche der ausseren Haarzellen der ersten Reihe zu den entsplechenden Flăchen der zweiten und darauf der dritten Reihe; worauf sie noch höher aufsteigen zu einigen Zellen in der Spitze der Schnecke.

L. Katzi , dessen Untersuchungen sich auf die Schnecke des Kaninchens, der Katze und Maus erstrecken, bestătigt im Wesentlichen die Beschreibung von Retzius. Nur in folgenden Punkten hatte er über die von diesem Forscher beschriebenen und genau localisirten spiralen Faserzüge eine etwas abweichende Meinung; nämlich Katz behauptete, dass der innerhalb der inneren Ifeilerzellen gelegene innerste Spiralzug, mit einem hier befindlichen Netz von Nervenfasern im Zusammenhang steht. Aus diesem Netzwerk sollen feine Fäserchen hervorgehen, welche zu dem unteren Ende der inneren Stäbchenzellen verlaufen, diese umgreifen und sich in 2-3 Fortsätze der inneren Stäbchenzellen einsenker. Dieser Autor fand ferner, dass die von dem bekannten spiralen Tunnelstrange nach aussen verlaufenden Fäserchen, in verschiedenen Höhen den Tunnel durchziehen. Iie am höchsten gelegenen ziehen $z$ wischen den äusseren Pfeilern hindurch direct zu dem innerenseits des von ihm als ,zangenbeckenförmige Gebilde" bezeichneten Theiles der Stützfasern der Deiters'schen Zellen, welches die ausseren Pfeilerzellen aufnimmt. Die tiefer verlaufenden radiären Fäserchen gelangen auf demselben Wege zur inneren Seite der Stützfasern selbst, und hier sieht man von ihrer Basis bis zu den zangenbeckenförmigen Gebilden hinter einander kleine Knöpfchen, welche wiederum Querschnitten solcher spiralen Nervenfaserzüge entsprechen, die aus der Umbiegung herantretender radiürer Fasern hervorgingen. Von der am zangenbeckenförmigen Gebilde gelegenen obersten spiralen Faser glaubte Katz sehr kurze Nervenfäserchen an das untere Ende der Corti'schen Zellen herantreten zu sehen.

Wie die Literatur über die Verlaufungsweise der Endfasern des Nervens cochleae, die wir oben kurz angeführt haben, zeigt, kann kein Zweifel darüber bestehen, dass jene Endfasern, welche die Autoren als die longitudinalen und transversalen oder als radiale und spirale Ziuge bezeichneten, dieselben Nervenfasern

1) I, Katz, Ueber die Endigung des Nervus cochlene im Corti'schen Organ. Archiv f. Ohrenheilk. Bd. XXIX. 
sind, welche nur wegen des bald radialen bald spiralen Verlaufs der Faser bald als radiale bald als spirale Züge unterschieden worden sind. Ferner steht die Thatsache fest, dass die Verlaufsweise der Endfasern des Nervens cochleae bei den verschiedenen 'Thieren ebenso verschieden ist und nach Ansicht der Autoren nicht einmal bei einem und demselben Thiere immer gleich ist.

Ich werde nunmehr, was ich iiber diese Beziehungen bei meinen Untersuchungen gefunden habe, im Folgenden darlegen. Nach meinen Beobachtungen werden die durch die Löcher der Habenula perforata herauskommenden Endnervenfasern in ihrem weiteren Verlauf, wie Boettcher, Waldeyer und Gottstein u. A. auch schon bemerkt haben, jedesmal von den Zellen aufgenommen. Sobald die Nervenfasern die Löcher verlassen haben, verbinden sie sich mit jenen Zellen, die ich schon oben als eigenthümliche Ganglienzellen angesehen und mit den subepithelialen Ganglienzellen der Retina verglichen habe. Von diesen Ganglienzellen gehen die Nervenfasern, die ich gewissermassen als Fortsätze dieser Zellen ansehe, theils nach den Zwischenräumen der inneren Pfeilerzellen, und anastomosirten in ihrem Verlaufe ebenfalls durch seitliche Fortsätze unter einander. Die Fasern, die nach Wald eyers Beschreibung von den Löchern der Habenula perforata aus direct durch die Körnerschicht hindurch nach den inmeren Haarzellen ziehen sollen, habe ich bisher nicht finden können. Ebenso ist mir auch das Vorhandensein des innersten spiralen /uges, den zuerst Hensen genau beschrieben und lietzius und Katz constatirt haben, ganz zweifelhaft. Wenn auch Retzius bei dem Kaninclen manchmal den scharf begrenzten Querschnitt dieses Zuges gesehen haben will, und dies der Angabe Boettchers gegenüber, „dass dies Niemand gelungen sei" besonders hervorhebt, so kann ich mich damit doch nicht einverstanden erklären, weil ich trotz der verschiedenen Methoden, besonders auch der gerade von Retzius angewendeten, nach der ich nicht weniger als 30 Kaninchen-Köpfe untersucht habe, doch nicmals den Querschnitt, den Retzius gezeichnet hat, sehen können. Ich finde weder auf dem Radialschnitte durch die Schnecke der verschiedenen von mir untersuchten Thiere jenen abgegrenzten Querschnitt dieses Zuges - während alle die anderen Spiralenzïge deutlich zu erkennen 
sind -, noch sind auf dem Horizontalschnitte der Schnecke bei Kaninchen, Katze, Hund, Meerschweinchen und Ratten irgend welche Spuren solcher spiralen Fäserchen zu sehen, die wie die anderen spiralen Züge continuirlich verlaufen. Es scheint allerdings auf dem Horizontalschnitte manchmal bei schwacher Vergrösserung, als ob der Spiralzug, der dicht ausserhalb der inneren Pfeilerzellen liegt, auch innenseits von denselben vorhanden wäre; aber durch genauere Untersuchung habe ich mich bei starker Vergrösserung jedesmal leicht von dem Irrthum überzeugen können.

Der spirale Zug, den Retzius den Tunnelstrang nennt, liegt auf dem Radialschnitte bei allen Sãugethieren, dicht an der Aussenseite der inneren Pfeilerzellen in ein Drittel der Höhe des Tunnelraumes. Sein Querschnitt ist gewöhnlich rund oder rundoval. Form und Grösse dieses Zuges ist aber nicht nur bei den verschiedenen Thieren etwas von einander verschieden, sondern auch in der Gegend der Schneckenwindung eines und desselben Thieres oft ungleich. Gewöhnlich ist dieser Spiralzug, wie schon viele Autoren richtig angegeben haben, ein ganz scharf begrenzter Strang; doch habe ich beim Hunde, manchmal auch beim Kaninchen, noch einige kleine Züge, die unterhalb des grossen Hauptzuges lagen, gesehen.

Von diesem Tunnelstrange gehen die sogenannten radialen Tunnelfasern nach aussen als. Diese Fasern laufen bei Kaninchen und Meerschweinchen gewöhnlich, ein kleines Bündelchen bildend, durch den Tunnelraum gerade nach aussen oder, indem sie sich etwas erheben, zu dem Spalte zwischen den äusseren I'feilerzellen. Nicht häufig laufen sie, wie bei anderen Thieren, besonders wie z. B. bei Hund und Katze, absteigend nach dem Tunnelboden oder nach der Fussplatte der ausseren Pfeilerzellen. Ferner findet sich bei Hund und Katze am Boden des Tunnels, wie Figur 3 u. 5 tbs. zeigt, ein spiraler Zug, den Retzius schon bei der Katze zuerst beschrieben hat. Dieser Zug ist gewöhnlich kleiner wie der Tunnelstrang und bildet einen accessorischen spiralen Zug.

Von diesen Spiralzügen laufen die radialen Fasserchen theils nach oben aussen durch die Zwischenspalten der äusseren Pfeilerzellen in $\mathrm{Nuels}$ Raum, theils gerade nach aussen durch den unteren Raum des Zwischenraumes der ausseren Pfeilerzellen in die Umgebung der Deiters'schen Zellen. 
Woher stammen nun diese spiralen Züge, die ich Tunnelbodenspiral/üge nennen will? I)ie radialen l'asern des Tunnelstranges, die wie ich kurz oben beschrieben habe, zu dem Boden des 'Tunnels absteigen, bilden ohne Zweifel zum Theil diese spiralen Zïge. Aber doch giebt es noch viele Fäserchen, die innenseits von den inneren I'feilerzellen, ohne Beziehung zu dem 'Tunnelstrange sich zu diesem Zuge vereinigen. Welcher von diesen beiden Faserzitgen den Hauptbestandtheil der 'Tunnelbodenspiralziige liefert, vermag ich hier nicht 'zu entscheiden. Was nun die Verhältnisse zwischen den radialen und spiralen Nervenfaserzuigen in 'Tumnelraum betrifft, so sind die Fasern, welche von der Innenseite der inneren Pfeilerzellen zwischen diese hindurch in dan Tumnelraum treten, niclit alle in dem Tunnelstrange vereinigt, sondern einige sind, wie ich oben bei Hund und Katze erwähnte, direct von der Innenseitè der inneren Pfeilerzellen aus, ohne lieziehung zu dem Tunnelstrange zu gewinnen, zu den Tunnelbodenspiralzügen verbunden. Aber es giebt kein radiales Fïserchen, das ohne einen dieser beiden Stränge zu berühren, direct zu den ausseren Pfeilerzellen hinliefe,

I)ie sogenannten Radialfasern des Tunnelraumes, die von dem Tunnelstrange in verschiedener Höhe durch den Tunnelraum nach den Zwischenspalten der ausseren Pfeilerzellen laufen, sind Fortsetzungsfasern der Nervenfasern des Tunnelraumstranges. Auf dem Horizontalschnitte durch die Schnecke liegt die Abgangsstelle der Radialfasern von dem Spiralzuge bald mehr dem Zwischenraume der inneren Pfeilerzellen, bald mehr der AussenHäche derselben Zellen genähert. Im ersten Falle scheint es, als ob die Radialfasern von den Zwischenraumen der inneren Ifeilerzellen aus direct nach aussen zugingen; doch halte ich dieses für wenig belangreich und meine, dass die durch die Zwischenräume der inneren Pfeilerzellell in den Tunnelraum heraustretenden Fasern, ohne spiralen Verlauf, nicht weiter radial laufen, und dass desshalb die Fasern, die ohne Beziehung mit dem Tunnelstrange zu dem Tunnelboden sich hinziehen, auch den Tunnelbodenspiralzug bilden.

Ferner finde ich auf Horizontalschnitten durch die Schnecke von Hund und Katze im Tunnelraume die von $\mathrm{Nuel}$ zuerst beobachteten Fasern, die von den Zwischenrüumen der inneren lfeilerzellen oder von dem 'Tumnelstrange aus nicht radial, sondern 
etwas in Spiralrichtung und absteigend zum Tunnelboden verlaufen. Diese Fasern laufen manchmal eine ziemlich weite Strecke im Tunnelraume, aber immer mehr nach aussen, zu der Fussplatte der äusseren Pfeilerzellen. Obwohl sie also von dem Tumnelbodenstrange verschieden zu sein scheinen, meine ich doch, dass alle als ihm zugehörig anzusehen sind.

Solche Nervenfasern, die wie Nu el schrieb, im Tunnelraume noch ihre Markscheide haben sollen, habe ich niemals angetroffen und muss desshalb an deren Existenz zweifeln.

Die in verschiedener Höhe durch die Zwischenräume der ausseren Pfeilerzellen nach au-sen ziehenden Fasern verbinden sich mit den lange bekannten äusseren Spiralzügen, die sich auf der Innenseite zwischen den unteren Enden der ausseren Haarzellen und den Deiter s'schen Zellen finden. Diese Züge bilden bei der Schnecke der Săugethiere gewöhnlich drei Reihen und beim Menschen, wie schon Retzius zuerst festgestellt hat, theilweise vier Reihen. Der Querschnitt dieser Züge ist feinkörnig und nicht scharf gegen die Umgebung abgegrenzt; bei gewöhnlichen Radialschnitten ist es schwer, diesen Zug von dem Zellkörper der Haarzelle und der Deit er s'schen Zelle zu unterscheiden. Ferner finden sich auf dem Radialschnitte durch die Schnecke an der Innenseite der Deiters'schen Zellen Querschnitte der Fasern und erscheinen hier als scharf abgegrenzte einreihig geordnete runde Punkte. Diese Punktreihen beginnen bei den Säugethieren oben am unteren Ende der ausseren Haarzellen und ziehen sich an der Innenseite der Deiters'schen Zellen in ziemlich regelmässigen Abständen nach der Basilarmembran zu. Die erste Reihe endigt auf der Membran, die zweite, deren Punktzahl geringer ist, jn einiger Entfernung von dieser, die dritte mit noch weniger Punkten in entsprechend grösserer Entfernung.

Diese Spiralzüge habe ich besonders bei Kaninchen und Hunden genau beobachtet. Bei dem Hunde sind, wie Figur 3 zeigt, die einreihigen Punkte in der ersten Reihe von oben nach unten bis zur Basilarmembrane in ganz regelmässigen Abständen geordnet; die zweite Punktreihe ist nur halb so lang als die erste Reihe und in der dritten finden sich nur 4--5 Punkte in einreihiger Anordnung. Unter den unteren Enden der ăusseren Haarzellen bilden die Punkte einen Haufen, der in der 
ersten Reihe deutlich sich mit den Radialfasern, welche durch den Tunnelraum herantreten, verbinden. An dieser Stelle besteht kein Unterschied zwischen den Querschnitten der ausseren Spiralstränge und der einreihigen Fasern, da beide Querschnitte nicht nur in ihrem Character ganz gleich sind, sondern auch manchmal einige Querschnittpunkte, die in der ersten Reihe unter dem ausseren Spiralzuge liegen, direct mit den Radialnervenfasern verbunden sind.

Bei den Präparaten, die ich durch Formolfixation von frischen Schnecken erwachsener Thiere und nach Doppelfärbung mit Hämatoxylin und Eosin gewonnen habe, ist das Bild, wie Figur 5 zeigt, ganz anders. Auf Radialschnitten sind die äusseren Spiralstränge nicht scharf abgegrenzt und die Punkte darin nicht ganz deutlich zu sehen. Auch sind die einreihigen Punkte an der Innenseite der Deiters'schen Zellen nicht continuirlich und doch hat jede Reibe ungefăhr die gleiche Zahl von Punkten. Diese Punkte finden sich besonders fein glanzend in den Theilen, wo die Zellkörper der Deiters'schen Zellen nicht körnig sind. Jede Reihe dieser Punkte hat einige grosse Punkte, die im oberen brittel der Reihe liegen und manchmal mit den Radialnervenfasern verbunden sind. Wenn man diese grossen Punkte bei stärkerer Vergrösserung genau beobachtet, bemerkt man leicht, dass sie aus vielen kleinen Punkten bestehen. Ferner finden sich zwischen den Hensen'schen Stützzellen bald einzelne, bald mehrere aneinander gereihte Punkte, die ganz gleichartig wie die sogenannten einreihigen Punkte erscheinen.

Beim erwachsenen Kaninchen ist das Bild meistens gleich dem beim erwachsenen Hunde; nur unter den einreihigen Punkten kommen bisweilen die dicken Querschnitte der vereinigten Fasern vor. Die drei I'unktreihen sind ebenso regelmässig geordnet und haben ungefihr die gleiche Anzahl von Punkten, nur sind diese etwas grösser als beim Hunde. Die erste Punktreihe reicht immer bis an die Basilarmembran, und die zweite und dritte bleiben nur etwas davon entfernt.

Ferner finde ich beim Kaninchen zwischen den Hensenschen Stützzellen viele deutliche Punkte, die manchmal durch feine Fasern mit den einreihigen l'unkten verbunden sind. Diese Fasern laufen in regelmässigen Abständen von einander, aber in 
den meisten Fällen nur über eine Zelle. Dieselben Fasern verbinden zuweilen auch die Punkte zweier verschiedenen Reihen und ebenso zwei Punkte zwischen den $\mathrm{Hensen}$ 'schen Zellen.

Was nun die einreihigen Punkte anbetrifft, so sind sie die Querschnitte der Fasern, die beim Horizontalschnitte an der Innenseite der D e i ter s 'schen Zellen regelmässig, wie Telegraphendrähte liegend, in spiraler Richtung verlaufen. In demselben Schnitte finden sich unterhalb der äusseren Haarzellen massenhafte Spiralfaserzüge, die den ausseren Spiralsträngen entsprechen. Diese Spiralfaserzüge verbinden sich mit den durch den Tunnelraum herantretenden Radialfasern und es ist daher zweifellos, dass sie von den Radialnervenfasern abstammen.

Woher stammen aber die einreihigen Spiralfaserzüge? Bei solchen Thieren, in deren Tunnelraume sogenannte Tunnelbodenspiralzüge vorhanden sind, müssen Fasern, die auf dem Tunnelboden nach aussen verlaufen, entsprechend den Drehungen der Spiralzüge auch Spiralzüge bilden. In der 'That habe ich beim Hunde gesehen, dass die Fasern von den Tunnelbodenzügen aus den Tunnelboden entlang zwischen den äusseren Pfeilerzellen hindurch in spiraler Richtung verlaufen. Bei den Thieren aber, bei denen keine Tunuelbodenspiralzüge vorhanden sind, wie zum Beispiel bei Kaninchen, ist es schwer den Ursprung dieser Züge zu erklären. Deshalb giebt es Autoren, die diese Züge nicht als Endnervenfasern gelten lassen. Ich halte aber mit Retzius und anderen Forschern daran fest, dass sie als Nervenfasern anzusehen sind. Denn ich habe in den Präparaten von der Schnecke des Kaninchens, die ich mit Fle m ming'scher Flüssigkeit fixirte und mit Hămateïn gefärbt hatte, gesehen, dass die einreihigen Spiralzüge allmählich nach oben verlaufen und schliesslich sich mit den sogenannten äusseren Spiralzügen vereinigen, oder unter diesen Zügen direct mit den durch den Tunnelraum herantretenden Radialfasern sich verbinden. Den weiteren Verlauf dieser Spiralzüge beschreibe ich in dem nächsten Abschnitte.

\section{Besondere Endigungsweise der Nervenfasern.}

Im Jahre 1854 schrieb Köllike r ${ }^{1}$ ) über die Endigungsweise des Nervus cochleae, dass die Ausbreitung des Schnecken-

1) Kölliker, Ueber die letzten Endigungen des Nervus cochleae und Function der Schnecke. Festschrift zum 50 jährigen Doctorjubiläum von F. Tiedemann, 1854. 
nervus nicht in der Scala tympani endige, sondern dass seine Fasern viel weiter durch die Löcher in der Lamina spiralis membranacea in die Scala vestibuli träten und hier in den Corti'schen Zahnen der zweiten Reihe, die er Corti'sche Fasern benannte, endeten. Diese Annahme von Kölliker fand bei anderen Autoren keine Zustimmung. Vielmehr behaupteten Boettcher ${ }^{1}$ ), Leydig ${ }^{2}$ ), M. Schultze ${ }^{3}$ ), dass die Endnervenfasern nicht in den Corti'schen Fasern endigen und dass die Corti'schen Fasern überhaupt gar nicht nervöser Natur seien. Köllike r ${ }^{4}$ ) selbst wurde in Folge weiterer Untersuchungen in seiner früheren Ansicht, nach welcher eine Verbindung der Nervenfasern mit den Corti'schen Fasern bestehen sollte, zweifelhaft, nachdem er im Vestibulum des Ochsen mit Sicherheit das Eindringen der Nervenenden in das Epithel der Nervenwarzen gesehen hatte.

Von dieser Zeit ab werden die Angaben der Autoren über die Endigungsweise der Endnervenfasern einheitlicher und übereinstimmender. Boettche $\mathrm{r}^{5}$ ) entdeckte nämlich zuerst, dass die blassen cylindrischen Fäden von den Löchern der Habenula perforata schräg nach oben und aussen hin in eine Reihe länglicher Ganglienzellen übergingen. Jiese Beobachtung bestätigte im nächsten Jahre I) eitersis, indem er zeigte, dass an der Innenseite des Bogens, der Pars membranosa zunăchst, eine Reihe cylindrischer cilientragender Zellen liegt, deren untere Spitze gewöhnlich mehr oder weniger lang ausgezogen ist. Obgleich Deiters eine gründliche Untersuchung über die Verlaufungsweise der Endnervenfasern angestellt hatte, konnte er doch über die Endigungsweise derselben damals noch nichts Genaueres mittheilen. Aber bald darauf wurde die Verbindung

1) Boettcher, Observatio microscopiae de ratione qua nervus cochleae marnmalium terminatur. Dorpati 1856.

2) F. Leydig, Lehrbuch der Histologie des Menschen und der Thiere. Frankfurt a. M. 1857.

$\left.{ }^{3}\right)$ M. Schultze, Ueber die Endigungsweise des Hörnerven im Labyrinth. Muller's Archiv. 1858.

4) Kölliker, Handbuch der Gewebelehre des Menschen, Dritte Auflage. Leipzig 1859.

5) Boettcher, Weitere Beiträge zur Anatomie der Schnecke. Virchow's Archiv. 1859. Bonn 1860 .

6) Deiters, Untersuchung über die Lamina spiralis membranacea 
der inneren Haarzellen mit den Nervenfasern von Kölliker ${ }^{1}$ ) und Middendorf ${ }^{2}$ ) beobachtet.

Den directen Uebergang der Endnervenfasern in die äusseren Haarzellen hat zuerst Emil Rosenberg ${ }^{3}$ ) gesehen. Er wies nach, dass die die Zwischenräume der äusseren Faserreihe passirenden Endnervenfasern in den Bereich der „äusseren Deckzellen" gelangen und geraden Weges zu den Corti'schen Zellen verlaufen, an deren unterem abgestumpften Ende sie endigten, indem je ein Fadchen an je einer Zelle mit dem Protoplasma des Zellkörpers verschmilat." Die Beobachtung, dass die Endnervenfasern direct mit den inneren und äusseren Haarzellen verbunden sind, wurde später von Boettcher ${ }^{4}$ ) und Winiwarter ${ }^{5}$ ) bestätigt. Ersterer glaubte damals allerdings, dass die ab- und aufsteigenden äusseren Haarzellen mit Endfäden des Nervus cochleae in Verbindung ständen.

Während in der Nachzeit die meisten Forscher sich mit dem blosen Nachweise von Verbindungen der Haarzellen mit den Endnervenfasern begnügten, gelang es He n sen ${ }^{6}$ ), in den äusseren Haarzellen eine bis dahin unbeachtete eigenthümliche Bildung zu entdecken, nămlich ovale Kapseln, welche eine glänzende, in Spiraltouren verlaufende Streifung zeigten. Hensen glaubte, dass diese Kapseln Nervenendapparate sein, welche dazu dienten, die Druckwahrnehmung zu vermitteln.

Nachdem aber eine Reihe angesehener Forscher, unter ihnen Gottstein ${ }^{7}$, Waldeyer ${ }^{8}$, $\mathrm{Nuel}^{9}$ ) und Boettcher ${ }^{10}$ ),

2) Kölliker, Handbuch der Gewebelehre des Menschen. Funfte Auflage. Leipzig 1867 .

2) M id d en d o r f, Monatschrift für Ohrenheilkunde. 1868.

3) E. Rosenberg, Untersuchungen uber Entwickelung des Canalis cochleoris der Säugethiere; Doctorats-Dissertation. Dorpat 1868.

4) Boettcher, Ueber Entwickelung und Bau des Gehörlabyrinthes nach Untersuchungen an Säugethieren. Dresden 1869.

5) Winiwarter, Untersuchnng ủber die Gehörschnecke der Säugethiere. 1870.

6) Hensen, Ueber Boettchers Entwickelung und Bau des Gehörlabyrinthes nach eigenen Untersuchungen. Archiv f. Ohrenheilkunde. 1871.

3) Gottstein, l. c.

8) Waldeyer, 1. c.

9) N u e I, Beitrag zur Kenntniss der Säugethiereschnecke. Archiv f. mikroskop. Anatomie. 1872.

1i) B o e t t c h e r, Kritische Bemerkung und neue Beiträge zur Literatur der Gehörschnecke. Dorpat. 1872. 
Ueber den peripheren Verlauf und die Endigung des Nervus cocbleae. 169

gerade die Haarzellen als die Endnervenzellen proklamirt und die Verschmelzung der Endnervenfasern mit den Zellkörpern betont hatten, gab Hensen ${ }^{1}$ zu, dass er zwar nach seinen Befunden nicht unbedingt der Beschrcibung Boettchers und Gottsteins zustimmen könne, es jedoch subjectiv für höchst wahrscheinlich halte, dass der Endverlauf so sei, wie sie angegeben hätten.

Obgleich also über die Verknüpfung von Haarzellen und Nervenendfasern, unter den Forschern fast allgemeine Uebereinstimmung herrschte, weigerte sich doch Retzius ${ }^{2}$ ), sich dieser Ansicht anzuschliessen, weil er überhaupt damals über diesen I'unkt noch nicht genügende Untersuchungen angestellt hatte. Er schrieb nämlich über die inneren Haarzellen, „theils gehen einzelne Fäserchen an den Haarzellen empor und umstricken ihre unteren Theile sowie die sie umgebenden Epithelzellen“. Er fragte ferner: "Wie endigen nun diese Fasern? Hicr bleibt eben die grösste Lücke in unserer Kenntniss vom feineren Bau des Gehörorgans. Ich habe mich vielfach bemiiht, diese Lücke auszufüllen, bisher aber fast vergebens. So viel ist jedoch sicher, dass die unteren Enden der äusseren Haarzellen die oberen Fasern der Spiralzüge berühren und ihnen sogar anhaften; einen directen Uebergang der Nervenfasern in die Haarzellen sah ich aber nie; nie sah ich die von $\mathrm{Nu}$ el beschriebenen, naeh oben zu den Haarzellenenden hin emporsteigenden Nervenfasern. IDiese Hauptfrage der Histologie des Gehörorganes der Singethiere muss meiner Ansicht nach noch als unbeantwortet betrachtet werden".

Nach Retzius veröffentlichte Tafan $i^{3}$ ) und Kat $z^{4}$ ) ganz ähnliche Beobachtungen, dass nämlich von den obersten Spiralfasern sehr kurze Nervenfäserchen an das untere Ende der Corti'schen \%elle heraustreten und auch in den Körper der Deiters'schen Zelle schr kurze und feine Nervenfasern aus den Spiralfasern hineinstrahlen.

1) Hen s e n, Besprechung. Archiv f. Ohrenheilkunde. 1873.

2) Retzius, l. c.

3) Tafani, l'organe de Corti chez les sings. Archiv ital. de biologic. 1885 .

4) Katz, Ucber die Endigung des Nervus cochleac im Corti'schen Organ. Archiv f. Ohrenheilliunde. Bd. XXIX. 
Wie schon oben erwähnt, zeigten die Resultate der verschiedenen Forscher, dass es nicht möglich sei, weitere Kenntnisse über die Endigungsweise des Nervus cochleae zu gewinnen, solange keine vollkommeren Untersuchungsmethoden vorhanden waren. Ein Fortschritt in der Forschung trat erst ein, nachdem die von Golgi entdeckte Versilberungsmethode der Nervenfasern, die auch in der Kenntniss der allgemeinen Neurologie einen vollständigen Umschwung hervorgerufen hatte, auch zur Untersuchung der Endigungsweise des Nervus acusticus angewendet wurde.

Ka ise ${ }^{1}$ ), welcher zuerst nach der Golgi'schen Methode das Epithel der Crista und Mucula acustica untersuchte, wies nach, dass die Achsencylinder sich nach ihrem Eintritte in das Epithel nicht in einzelne Fibrillen auflösen, sondern, dass ein jeder von ihnen ungetheilt zum Grunde einer Haarzelle vordringt und dieselbe mit einem kelchähnlichen Ende umfasst; dieser nervöse Kelch besteht aus derselben hyalinen Grundsubstanz wie der Achsencylinder und die darin eingebetteten kraftigen, stark lichtbrechenden Körnchen.

Durch Anwendung derselben Methode gelangte Retzius s'), der sich in der aller letzten Zeit sehr um die Klarstellung der peripherischen Endigungsweise des Gchörnervens bemüht hatte, auf Grund seiner Untersuchungen bei der Maus und dem Hühnchen zu vollständig neuen Angaben, die er auch bei Kaninchenembryonen bestätigen konnte. Retzius wies zuerst darauf hin, dass in den verschiedenen Sinnesorganen auch die Endigungsweise der Nerven sich etwas verschieden verhalte. Er hatte auch im Gehörorgan der Vögel und Säugethiere und zwar sowohl in den Maculae und Cristae acusticae erkannt, dass die Haarzellen des Epithels nicht, - wie etwa im Riechorgane die Riechzellen mit dem Nervus olfactoruis, - direct mit den Nervenfasern des Acusticus zusammenhängen, resp. nicht die Ursprungszellen dieser Nervenfasern sind, sondern nur von denselben intim umsponnen werden. Dagegen stellte er die bipolaren Ganglienzellen als den Riechzellen des Riechorgans gleichwertig dar.

1) $\mathrm{K}$ a i s e r, Das Epithel der Crista und Maculae acusticae. Archiv f. Ohrenheilkunde. 1891.

$\left.{ }^{2}\right)$ Retzius, Die peripherische Endigungsweise der Gehörnerven. Verh. der anat. Ges. Wien. 1892. 
Retzi us behauptete also, dass die Haarzellen des Gehörorgans keine wahren Nervenzellen seien, wie die Riechzellen des Riechorgans, und dass sie daher auch nicht den letzteren gleichwerthig sein könnten, sondern viel mehr als eine Art „indirecter Sinneszellen" aufgefasst werden müssten.

Diese neue Auffassung von Retzius, welche er den alten, schon vor langer Zeit von vielen Forschern constatirten Befunden entgegenstellte, wurde im nächsten Jahre von Lenhossek ${ }^{1}$ ) bestätigt. Dieser untersuchte nach derselben Methode und zwar dieselben Thiere wie Retzius, hauptsächlich an der Macula acustica sacculi, und erklärte, dass jede der Fasern, die zur Zone der Haarzellen emporsteige, sich hier zuerst zu einem kleinen Knötchen verdicke, dann sich der Basis einer Haarzelle anlege und sich in 3-4 Aeste theile. Diese ziehen rechtwinkelig zu dem Verlauf der zutretenden Faserstränge horizontal, d. b. mit der Oberfläche des Epithels parallel unter einer Anzahl von Haarzellen hin, in enger Berührung mit deren Basis, um schliessllch mit emporgebogenen Spitzen frei zu endigen. Unterwegs geben sic einige aufsteigende Aestchen $a b$, die an der SeitenHaiche der Haarzellen senkrecht emporziehen, ohne aber jemals die OberHache des Epithels zu erreichen.

Während diese drei Forscher auf Grund der Golgi'schen Methode zu neuen, wenn auch unter einander abweichenden Resultaten gelangt waren, ging Ayers ${ }^{2}$ ) andererseits bei Anwendung derselben Methodik zu alten Anschauungen zurück. Er kam bei seinen Untersuchungen zu folgenden Schlusssätzen; a) dass die Haarzellen und ihre anhängenden Ganglienzellen eine einzige morphologische Einheit bilden - ein akustısches Element - welche zwischen obertlachlichen und centralen Punkten vermittelt; b) dass keine fundamentale Verschiedenheit zwischen acustischen und olfaktorischen Elementen existirt; c) dass die sogenamnten "Spiralfasern" nur kurze Strecken von Radialfasern sind, welche ilı Ziel nach umlaufendem Wege erreichen; d) dass alle Fasern des Nervus acusticus, soweit es sicher dargestellt ist, ihren Ursprung in den Haarzellen nehmen.

2) L e nhos sek, Nervenendigung im Gehörorgane. Vor. der anat. (xes, 1893.

2) A y e rs, Ueber peripherisches Verhalten der Gehörnerven und den Werth der Haarzellen des Gehörorgans. Anat. Anz. VIII. Jahrg. 
Selbst mit der Golgi'schen Methode schien es demnach schwer zu sein, die Endigungsweise der Gehörnerven zu ermitteln. Desshalb nahm Ni e ma ck ${ }^{2}$ ) seine Zuflucht zu Ehr lichs Methylenblaumethode, und veröffentlichte auf Grund derselben Befunde, die er mit dieser Methode bei Kaninchen gewonnen hatte. Vier Jahre später theilte dann $\mathrm{Krause}^{3}$ ) in der anatomischen Gesellschaft in Berlin mit, dass die Zahl der einstrahlenden Nervenfasern mit der Vergrösserung der Nervenendstelle wachse. Zunächst gewahre man, dass das freie Ende der Faser sich etwas verdicke, in die Breite wachse, und sich flach, becher- oder kelchförmig aushöhle. Aus diesem verbreiterten Ende schössen dann zahlreiche Fabrillen hervor, welche den Körper der Epithelzelle eng umspönnen; in anderen Fällen sei die Verdickung des Faserendes von vornherein sehr wenig ausgesprochen; hier sehe man an ihrer Stelle 3-5 kurze, stark gekrümmte, relativ kräftige Zweige aus der Faser hervorsprossen, welche das untere Ende der Epithelzelle klauenförmig eng umklammern. Häufig erschienen dann nach den Angaben von Krause, bald schwächer, bald stärker blau gefärbte Epithelzellen, und man könne ganz allgemein sagen, dass sich um so mehr Zellen fänden, je ausgiebiger und präciser die Färbung der Nervenfasern gelungen sei. Dieser Umstand ermögliche es nun auch zu erkemnen, wie ausserordentlich eng und innig die Umspinnung oder Umklammerung der Epithelzellen durch die aus der hinzutretenden Faser hervorsprossenden Fibrillen sei. Krause erklärte zuletzt; obwohl seine Resultate in dieser Beziehung noch recht fragmentarische seien, so halte er es doch für angezeigt dieselben vorzuführen, da sie von den neueren Resultaten von Retzius und vor allem von denen von Lenhossek wesentlich abweichen und sich mehr den von Niemack und Kaiser ermittelten Beobachtungen näherten.

Die beiden genannten Methoden, welche in letzter Zeit von den Forschern zu der Untersuchung der Endigungsweise der Endnervenfasern des Nervus Acusticus angewendet worden sind, haben in der That die Kenntniss der Anatomie des Gehörorgans wesent-

2) Ni emack, Maculae und Cristae acusticac mit Ehrlich's Methylenblaumethode. Anat. Hefte. Abt. 1, Bd. 2. 1892.

3) Krause, Die Endigungsweise des Nervus acusticus im Gehörorgane. Verhandl. d. anat. Gesellsch. in Berlin. 1896. 
lich gefördert, ohme jedoch sie zum letzten Ziele gebracht zu haben. Es scheint mir sicher, dass die nach verschiedenen Methoden gewonnencen Befunde grade desshalb abweichen, und dass hingegen die nach ein und derselben Methode gewonnenen Resultate aus demselben (irunde mit einander übereinstimmen. I)esshalb liegt die Folgerung nahe, dass vielleicht die eine von beiden Methoden oder beide fehlerhaft seien. Es war darum auch unbedingt nöthig, eine neue Methode in Anwendung zu bringen, um die bisher mit jenen beiden Methoden gewomnenen Resultate auf ihre lichtigkeit hin prüfen zu können.

Nach vielfachen Bemühungen ist es mir geglückt, eine wie ich glaube, sichere und zweckentsprechende Methode zu finden. Es ist dies eine Farbungsmethode mit Haemateïn, welches vor mir bereits von $A$ pat hy auf das Nervensystem angewandt worden ist, indess in einer Weise, welche die unten beschriebenen neuen Gebilde nicht hervortreten lässt. Mit Hülfe meiner Methode gelang es mir, die durch die letzten beiden Methoden schon erzielten Befunde genauer zu untersuchen. Als besonderen Vorzug dieser Methode den anderen gegenüber hebe ich ausdrücklich hervor, diss man sie nicht nur zur Stückfürfung, sondern auch für einzelne schnitte verwenden kann, walurend man mit den beiden anderen Methoden, nur um den Nervus zu untersuchen, entweder das ganze lebende 'Thier oder das ganze Labyrinth in Behandlung nehmen muss. Ausserdem bieten diese beiden Methoden besondere technische Schwierigkeiten, die sich bei unserer Färbungsmethode mit Haemateïn nicht finden. Nur muss man vorher das Material zur Untersuchıng ganz vollständig fixiren. Hierzu habe ich formol oder Formolosmium als besonders tauglich erkannt. Fïr den ersten Fall empfehle ich, das Material mit Formolwasserlösung allmählich zu fixiren, und bei Thieren von der Grösse des Kaninchens und der Katze vor dem lixiren zuerst den Steigbügel aus der Fenestra ovalis ganz vorsichtig herauszunehmen und dann auch die lienestra rotunda zu offinen, ohne jedoch die Wand des Ductus cochleae zu verlotzen. Nach dieser Vorbereitung legt man das l'rüparat zuerst in 1 proc. Formolwasserlösung, und macht dann nach je 24 Stunden die Lösung immer um 1 Proc. stärker, bis sie am vierten 'Tage 4 proc. ist, und lisst das Object in dieser 4 proc. Lösung einige Tage liegen. Ihas fixirte Präparat bringt man ohne vorheriges Auswaschen direct in 70 proc. Alcohol, lässt es darin 
3 Tage liegen, bei ein- resp. mehrmaliger Erneuerung des Alcohols, und legt es dann noch 2 Tage in 96 proc. Alcohol. Dann folgt die Entkalkung; zu dieser gebraucht man am zweckmässigsten nach der Vorschrift von Thoma 10-20proc. SalpetersäureAlcohol (96 Proc.), welcher einen Tag um den andern gewechselt werden muss. Nachdem die Säure mit Calcium carbonicum gräcipirt gesättigten 96 proc. Alcohol ungefähr 8 Tage lang neutralisirt ist, wird das Präparat noch einmal mit 96 proc. Alcohol ausgewaschen, weiter nach bekannter Regel in Celloidin oder Paraffin eingebettet und in $15-8, "$ dicke Serienschnitte zerlegt. Diese Schnitte kann man theils zur Untersuchung der allgemeinen Structur des ganzen Labyrinthes, theils zur speciellen Untersuchung der Verlaufsweise und Endigungsweise der Endnervenfasern verwenden. Unsere Methode gewährt also den besonderen Vorthcil, dass man an einem und demselben Materiale die ganze Structur des Organs beobachten kann. Sie ist deshalb auch für pathologische Untersuchungen im hohen Grade zweckmässig.

Die Färbstofflösung von Haemateïn habe ich aus käuflichem Haemateïn in folgender Zusammensetzung bereitet.

$\begin{array}{rr}\text { Haemateïn. . } & 1,0 \\ 70 \text { proc. Alcohol } & 100,0 .\end{array}$

In dieser Lösung liess ich den Schnitt wenigstens 24 Stunden, aber nicht länger als 40 Stunden liegen. Nachdem er einmal mit destillirtem Wasser gewaschen war, blieb er noch weiter nach Erneuerung $4-5$ Stunden darin liegen. Da in dem Wasser der Farbstoff ein wenig reducirt wird, so gewinnt der Schnitt eine hellbraune oder etwas grünbraune Färbung. Dann habe ich den Schnitt 20-24 Stunden in gewöhnliches Wasser hinein gethan, und zwar so, dass er von einer ziemlichen Menge Wassers bedeckt war und tief unten in dem Wasser lag. Nun wurde die Schnittfarbe mehr dunkelbraun oder schwarzbraun. Aus dem gewöhnlichen Wasser brachte ich ihn direct in 96proc. oder absoluten Alcohol und weiter in Carbol-Xylollösung und schloss ihn mit Canadabalsam ein.

Nach dieser Methode habe ich die Endigungsweise der Endnervenfaser hauptsächlich bei dem Kaninchen untersucht, und in dem Schnitte Einzelheiten gefunden, welche durch die gewöhnlichen Färbungsmethoden nicht zu entdecken waren. In solchen Schnittpräparaten zeigt das untere Ende der äusseren Haarzelle, wo es sich mit dem Zellkörper der Deiters'schen Zelle und 
mit dem äusseren Spiralnervenzuge berührt, niemals eine scharfe Grenze. Früher glaubten deswegen einige Autoren, dass die Corti'sche Zelle und Deiters'sche Zelle Zwillingszellen seien. Nach meiner Färbungsmethode erscheint in dieser Gegend dicht unter dem Kern der Haarzellen ein neues Gebilde, welches etwa kelchförmig gestaltet, sich besonders stark farbt und von der Umgebung ganz scharf abgrenzbar ist. Bei starkerer Vergrösserung zeigt es keine homogene Structur, sondern ist mehr körnig. Dieses k e l chförmig e Gebil de umfasst mit seiner Oeffinung das untere Ende der Haarzellen. Die Frage, ob hier die Haarzellen durch das kelchförmige Gebilde umsponnen oder umklammert sind, oder ob es mit dem Zellkörper verschmolzen ist, muss nach meiner Untersuchung dahin entschieden werden, dass es mit dem Zellkörper vereinigt ist, resp. einen Endtheil der Haarzellen bildet. Denn, obwohl zwischen dem Kern der Haarzellen und dem Boden des kelchförmigen Gebildes ein sichelförmiger Raum, wie Fig. $6 \mathrm{~kb}$. zeigt, sich befindet, so ist doch dieser Raum nicht leer, sondern mit dem Zellprotoplasma ganz erfüllt, und der Rand des kelchförmigen Gebildes geht ohne scharfe Grenze in die Oberfläche der Haarzellen über. Von der am unteren Ende befindlichen Spitze des kelchförmigen Gebildes setzt sich ein kurzes Fädchen fort, welches manchmal deutlich mit dem ausseren Spiralnervenzuge verbunden ist. Desshalb stellt nach meiner Ansicht das kelchförmige Gebilde eine eigenthümliche Structur des unteren Endes der Haarzellen dar. Ich finde keinen wesentlichen Unterschied zwischen diesen Zellen und den Riechzellen des Riechorgans. Auch bei den Riechzellen findet sich an der Stelle, wo die einzelnen Fasern des Nervus olfactorius entspringen, ein ähnliches Gebilde. Es ist mir darum zweifellos, dass die Endnervenfasern in den Haarzellen endigen, resp. dass der Nervus cochleae von den Haarzellen stammt, gerade wie der Nervus alfactorius von den Riechzellen.

Die Zweigfasern, die Krause durch die Methylenblaumethode von der (iegend des kelchförmigen Gebildes ab auf der Oberfläche der Zellkörper der Haarzellen emporsteigen sah, habe ich niemals bemerken können; vielleicht sind sie ein Kunstproduct seiner Vorbehandlungsmethode. Ebenso habe ich die Aestchen von Nerven, die nach Lenhosseks Beschreibung an der Seitenfläche der Haarzellen senkrecht emporziehen sollen, olne aber 
jemals die Oberfläche des Epithels zu erreichen, beim Cortischen Organ niemals getroffen.

Ferner habe ich mit dieser Methode die sogenannten einreihigen Spiralnervenzüge genau beobachtet, und es ist mir im hohem Grade zweifelhaft geworden, ob diese Fasern, wie die Nervenfasern der äusseren Spiralnervenzüge, auch in den Haarzellen endigen, weil ich ganz dieselben Fasern ausserhalb der äussersten Reihe der Deiter's'schen Zellen unter den Hensenschen Stützzellen bemerkt habe. Hier verlaufen die Fasern nicht ganz in Spiralrichtung, sondern nur in regelmässigen Abständen von einander in verschiedener Richtung, und verbinden sich recht oft mit den einreiligen Spiralnervenzügen. Wie sie nun aber in den Hensen'schen Stützzellen endigen und was für eine specielle Bedeutung sie haben, vermag ich zur Zeit nicht zu sagen, weil meine Untersuchungen hierüber noch nicht vollständig zum Abschluss gebracht sind.

Als ich diese Arbeit vollendet hatte, veröffentlichte G. Retzi us seine neue Untersuchung, „Zur Kenntniss der Gehörschnecke". (Biologische Untersuchungen. Neve Folge Bd. IX). Darin schrieb er, dass er mit Hilfe der Heidenhain'schen Eisen-Hämatoxylinmethode dicht unter dem Ende der äusseren Haarzelle in dem Deiter'schen Zellkörper einen Körnchenkörper gefunden habe. Vielleicht ist dieser nichts anders, als das Gebilde, das ich durch meine Hämateïnfärbung gefunden habe, das aber, wie ich schon oben genau geschrieben habe, nach meiner Beobachtung, nicht zu der Deiters'schen Zelle sondern zur äusseren Haarzelle gehört. Ich will hierbei noch besonders darauf aufmerksam machen, dass die Spitze dieses becherförmigen Gebildes immer nach dem ausseren Spiralnervenzuge gerichtet ist und manchmal damit verbunden erscheint. Auch möchte ich ebenfalls hier noch hervorheben, dass ich weder an der Umgebung dieses Gebildes noch am unteren Ende der iusseren Haarzellen eine Spur von Nervenfasern bemerkt habe, die nach Retzius u. A. den Zellkörper der Haarzellen umspinnend vorhanden sein müssten, wăhrend dagegen meine Färbungsmethode sehr feine Fädchen der anderen bekannten Spiralfasern ganz deutlich zu sehen sind. 
Ueber den peripheren Verlauf und die Endigung des Nervus cochleae. 177

\section{Die Schlusssäitze.}

A. Im sogenannten „Epitheldreiecke" der Schnecke von Säugethieren finde ich ausser den inneren Haarzellen noch drei verschiedene Zellen: 1. Epithelzellen, die den Epithelzellen des sulcus spiralis internus entsprechen. 2. Die sogenannten inneren Stützzellen, die einen länglich ovalen Kern haben. 3. Eigenthümliche Ganglienzellen, die mit den Fasern des Nervus cochleae verbunden sind.

13. Auf der Innenseite der inneren Pfeilerzellen resp. im sogenannten Epitheldreiecke finde ich bei Kaninchen, Meerschweinchen und Hunden keine Spiralfasern, im Unterschied zu den Angaben von Hensen. Retzius und Katz u. A.

C. Die Endnervenfasern, die durch die Zwischenräume der inneren Ifeilerzellen hindurch treten, laufen nach meiner Ansicht alle eine Strecke weit in spiraler Richtung. Dadurch allein entsteht der 'Tumnel- und 'Tunnelboden-Spiralzug.

I). Bei Hund und Katze und auch beim Menschen finden sich sogenamte 'Tumnelbodenspiralziige, die Retzi us zuerst beschrieben hat. Aber sie fehlen nach meiner Beobachtung nicht nur bei Kaninchen und Meerschweinchen sondern auch bei Ratte, Maus und Schaf.

E. Iie Hämateïnfärbungsmethode bietet bei der Untersuchung der peripheren Endfasern des Nervus cochleae manche Vortheile vor der Golgi'schen Methode und Eh rlich's Methylenblaumethode dar.

F. Iie Endfasern des Nervus cochleae, welche zu den Haarzellen treten, gehen je eine in ein bisher noch von Niemand beschriebenes, an dem unteren Ende der Haarzellen befindliches Gebilde von kelchförmiger Gestalt über, welche sich als einen Theil der Haarzellen betrachte.

G. Nach meiner Beobachtung sind die äusseren Spiralnervenfasern nur mit den Spitzen jener kelchförmigen Gebilde verbunden und steigt kein Fädchen von diesen Nerven zur Umgebung des oberen Theiles der Haarzellen empor.

H. Hinsichtlich des feineren Baues verhalten sich die Haarzellen des Gehörorgans wie die Riechzellen des Riechorgans, wie schon frühere Autoren angenommen haben. 
I. Die sogenannten einreihigen Spiralnervenzüge endigen nicht alle in den Haarzellen, sondern sie laufen zum Theil unter den Hensen'schen Stützzellen wejter.

\section{Figurenerklärung der Tafel VI.}

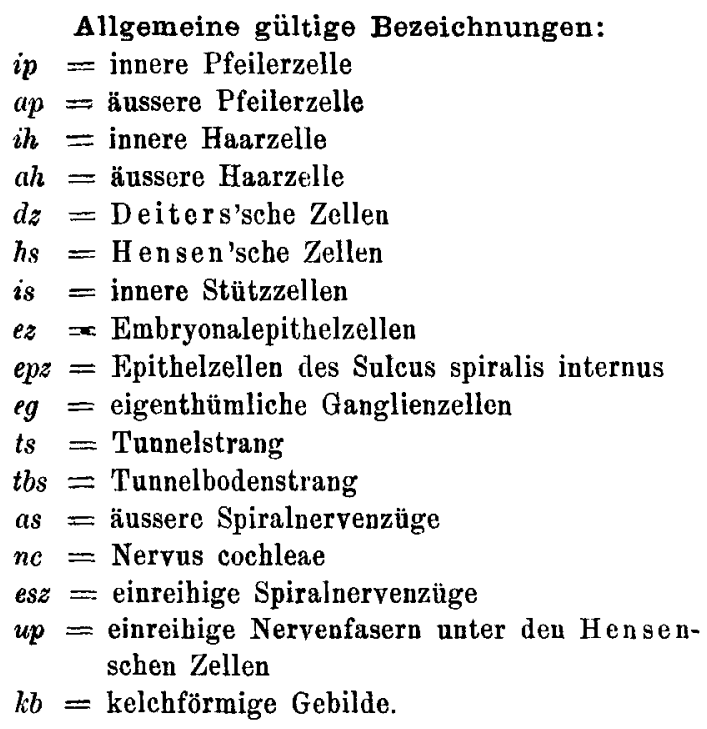

Fig. 1. Radialschnitt der zweiten Windung eines $8 \mathrm{~cm}$ langen Kaninchenembryo. Hämatoxylin und Carmin Doppelfärbung. Zeiss Apochr. Obj. E. Ok. 2.

Fig. 2. Radialschnitt der zweiten Windung eines zwei Tage alten Kaninchens, Mit Formol fixirt. Hämatoxylin und Eosin doppelt gefärbt. Zeiss Apochr. Obj. E. Ok. 2.

Fig. 3. Radialschnitt der zweiten Windung eines zehn Tage alten Hundes. Mit Osminmsäure fixirt. Hämatoxylin Färbung. Zeiss Apochr. Obj. E. Ok. 2.

Fig. 4. Radialschnitt der ersten Windung eines erwachsenen Kaninchens. Mit Formol allmählich fixirt. Hämatoxylin Eosin doppelt gefärbt. Zeiss A pochr. Obj. E. Ok. 2.

Fig. 5. Radialschnitt der zweiten Windung eines erwachsenen Hundes. Mit Formol fixirt. Hämatoxylin Carmin doppelt gefärbt. Zeiss Apochr. Obj. E. Ok. 2.

Fig 6. Radialschnitt der zweiten Windung eines erwachsenen Kaninchens. Mit Formol allmählich fixirt; mit Hämatëin gefärbt. Zeiss Apoch. E. Ok. 4 . 
Ueber den peripheren Verlauf und die Endigung des Nervus cochlcae. 179

\section{Lebenslauf.}

Ich Jchita (Kazuta) Kishi wurde geboren am 28 August 1874 zu Okayamaken (Japan). Ich besuchte das Gymnasium zu Okayama, woselbst ich im September 1893 das Zeugniss der Reife erhielt. Dann studirte ich an der kaiserl. medicinischen Hochschule No. 3 und bestand am 25. November 1897 daselbst mein Staatsexamen.

Vom 1. April 1898 an war ich als Assistenzarzt an einer Anstalt für Ohren-, Nasen- und Halskrankheiten zu Tokyo thătig. Am 13. Januar 1900 verliess ich meine Heimath, um in Europa weitere Studien in der Otologie zu machen. Seit meiner Ankunft in Europa studirte ich bis jetzt drei Semester auf der Universität Halle a. d. S. und bestand daselbst meine Fakultätsprüfung am 14. Juni 1901.

Wahrend meiner Studienzeit besuchte ich die Vorlesungen folgender Herren:

In Japan:

B. Adachi, S. Araki, T. Araki, H. Date, S. Furuhawa, Z. Jnoue, M Jnoue, F. Katsurada, S. Kumagai, K. Sakata, K. Takahashi, S. Tsuge, S. Yoshimura.

In Halle:

C. Fränkel, C. Grunert, E. Mehnert, W. Roux, H. Schwartze. 